\title{
INOVAÇÕES TECNOLÓGICAS NO USO DO PRAZIQUANTEL PARA O TRATAMENTO DA ESQUISTOSSOMOSE
}

\author{
L. P. SIQUEIRA ${ }^{1}$, E. O. SILVA ${ }^{1}$, V. A. W. SALES ${ }^{1}$, I. N. G. BARBOSA, ${ }^{1}$ C. S. B. \\ AGUILERA $^{1}$, D. A. F. FONTES ${ }^{2}$, T. R. R. TIMOTEO ${ }^{1}$, J.F.B. RODRIGUES ${ }^{1}$, L. A. \\ ROLIM $^{3}$, R.M.F. SILVA ${ }^{1}$, P.J. ROLIM NETO ${ }^{1}$
}

${ }^{1}$ Universidade Federal de Pernambuco, Centro de Ciências Biológicas, Departamento de Ciências Farmacêuticas, Laboratório de Tecnologia dos Medicamentos

${ }^{2}$ Faculdades Integradas da Vitória de Santo Antão

${ }^{3}$ Universidade do Vale de São Francisco, Central de Análise de Fármacos, Medicamentos e Alimentos

E-mail para contato: emersondeolliveira@gmail.com

RESUMO - A esquistossomose é uma doença causada pelo trematódeo intravascular Schistosoma mansoni e está associada à pobreza, condições precárias de higiene e baixo desenvolvimento econômico, com maior prevalência em áreas rurais e litorâneas. De acordo com a Organização Mundial de Saúde, estima-se que aproximadamente 240 milhões de pessoas estejam infectadas com o esquistossomo, sendo portadoras da forma aguda ou crônica da doença. A farmacoterapia utilizada no tratamento da esquistossomose apresenta algumas desvantagens devido à sua baixa eficácia e segurança. O praziquantel e a oxaminiquine são os fármacos de escolha para tratamento da esquistossomose. O praziquantel age em um espectro maior de parasitas do que a oxaminiquine, além disso, possui baixa toxicidade quando comparado a outros fármacos análogos. O Sistema de Classificação Biofarmacêutica (SCB) é utilizado para o desenvolvimento de fármacos e permite a determinação de um perfil farmacológico de novas drogas para prever possíveis obstáculos durante a absorção. Uma alternativa para driblar a baixa solubilidade do fármaco é a utilização de polímeros e complexos de inclusão, como as ciclodextrinas. Diversos estudos são relatados na literatura, com novas formas farmacêuticas a partir do praziquantel para melhora da sua solubilidade e consequente maior segurança e eficácia terapêutica.

Palavras Chave: Praziquantel, inovações tecnológicas, indústria farmacêutica.

ABSTRACT - Schistosomiasis is a disease caused by the intravascular trematosis Schistosoma mansoni and is associated with poverty, precarious hygiene conditions and low economic development, with greater prevalence in rural and coastal areas. According to the World Health Organization, an estimated 240 million people are infected with the schistosome, being carriers of the acute or chronic form of the disease. Pharmacotherapy used in the treatment of schistosomiasis presents some disadvantages due to its low efficacy and safety. Praziquantel and oxaminiquine are the drugs of choice for the treatment of schistosomiasis. Praziquantel acts in a larger spectrum of parasites than oxaminiquine, and has low toxicity when compared to other analogous drugs. The 
Biopharmaceutical Classification System (SCB) is used for the development of drugs and also allows the determination of a pharmacological profile of new drugs to predict possible barriers during absorption. An alternative to overcome the low solubility of the drug is the use of polymers and inclusion complexes, such as cyclodextrins. Several studies are reported in the literature, with new pharmaceutical forms from praziquantel to improve its solubility and consequent greater safety and therapeutic efficacy.

Keywords: Praziquantel, technological innovations, pharmaceutical industry.

\section{INTRODUÇÃO}

As doenças tropicais negligenciadas se prevalecem em condições de pobreza e contribuem para a manutenção da desigualdade social, sendo um grande obstáculo ao desenvolvimento de um país. A esquistossomose constitui um grande problema de saúde pública, sendo uma das doenças tropicais negligenciadas mais prevalentes, afetando mais de 200 milhões de pessoas em 78 países, sendo o Brasil a região mais endêmica nas Américas. Trata-se de uma parasitose causada pelo Schistossoma mansoni, cujas formas adultas habitam em vários locais dos vasos mesentéricos do hospedeiro vertebrado. Inicialmente, a doença é assintomática, podendo evoluir para formas clínicas graves e levar o paciente a óbito (SILVA et al., 2017).

O praziquantel (PQZ) é o fármaco anti-helmíntico de escolha para prevenção e tratamento da esquistossomose por sua eficácia contra todas as espécies de Schistosoma que afetam adultos e crianças. Seu mecanismo de ação acontece devido a inibição da bomba $\mathrm{Na}+$ e $\mathrm{K}+$ dos esquistossomos, aumentando a permeabilidade da membrana do helminto a cátions monovalentes e divalentes, principalmente ao cálcio, gerando uma intensificação da atividade muscular, seguida por contração e paralisia espática. (SILVA, 2012).

Por ser um medicamento eficaz, seguro e baixo custo, a Organização Mundial da Saúde considera que o praziquantel é uma droga essencial. Contudo, de acordo com o Sistema de Classificação Biofarmacêutica, o PQZ é um medicamento que pertence à classe II, possuindo uma baixa solubilidade. Isso prejudica o processo de dissolução, um fator limitante para a absorção, e consequentemente a sua biodisponibilidade. Diante dessa problemática, estudos na literatura propõe incremento da solubilidade através da utilização de carreadores e polímeros capazes de melhorar a biodisponibilidade do praziquantel (CUGOVCAN et al., 2017; SILVA et al., 2012).

\section{MATERIAIS E MÉTODOS}

A construção do referido trabalho, foi embasada, a partir de um levantamento de dados, realizado em sites periódicos, incluindo como base o Science Direct, Scielo e Google acadêmico, no período de 2005 a 2017. A pesquisa por palavras em títulos e resumos foi realizada utilizando as seguintes entradas: praziquantel, inovação tecnológica, classificação biofarmacêutica, esquistossomose, doenças negligenciadas e indústria farmacêutica.

\section{RESULTADOS E DISCUSSÃO}

Mesmo com poucos investimentos por parte das indústrias farmacêuticas na inovação tecnológica e otimização da terapia com o Praziquantel (PZQ), há estudos descritos na literatura que abordam o desenvolvimento de novas formulações empregando excipientes inteligentes na tentativa de melhorar as características biofarmacêuticas do PZQ, no tocante a 
sua baixa solubilidade em água, característica que limita sua biodisponibilidade.

Dentre essas novas alternativas, a estratégia nanotecnológica vem sendo empregada em todas as suas nanoescalas (lipossomas, nanoemulsões e nanopartículas), trazendo resultados impactantes na terapia esquistossomicida, por promover um tratamento mais otimizado no uso do PZQ, ao conseguir sanar as limitações biofarmacêuticas desse fármaco (DINORA, et al., 2005). Na encapsulação do PZQ em lipossomas, o fármaco além de ter sua solubilidade incrementada, ganha em estabilidade frente à acidez gástrica. $\mathrm{O}$ emprego de lipossomas tem a capacidade de promover aumento da eficácia da terapia com o PZQ em comparação com uma dose igual do mesmo princípio ativo em sua forma livre. Frezza et al. (2013), utilizou uma concentração de $300 \mathrm{mg} / \mathrm{kg}$ de PZQ encapsulado em lipossomas administrados em adultos com esquistossomose por um período de 45 dias, resultando na diminuição do número total de vermes em $68,8 \%$, no número de ovos no intestino em $79 \%$ e o número de granulomas hepáticos em 98,4\%, além de diminuir a contagem de ovos em 55,5\%.

Em estudo in vitro, Souza et al. (2014), ao incorporar o PZQ em nanopartículas lipídicas sólidas, observaram uma diminuição da absorção intestinal do fármaco, sugerindo que essa matriz nanotecnológica atue como um sistema reservatório, permitindo uma liberação controlada do PZQ no trato gastrointestinal. Nesse mesmo estudo, a encapsulação do PZQ veio a diminuir a sua citotoxicidade frente à linhagem celular de hepatoma humana HepG2 em comparação com a solução PZQ livre em uma concentração equivalente.

O uso de ciclodextrinas em suas variadas formas para complexar fármacos como o PZQ, está bem descrito na literatura com diversos estudos que comprovam o emprego desses excipientes na tentativa de incrementar a solubilidade aquosa desse ativo, com resultados excepcionais. Contudo, autores vem melhorando o emprego desse excipiente para a formação de complexos com o PZQ, na adição de adjuvantes poliméricos, otimizando a formação dos sistemas (Ciclodextrina/PZQ) (RODRIGUES et al. 2011). Mourão et al. (2016), avaliou o efeito do Hidroxiproprilmetilcelulose (HPMC), na complexação do PZQ com betaciclodextrina $(\beta C D)$, verificando uma melhora na complexação do fármaco, por ocasionar um incremento na solubilidade intrínseca do ativo. Nesse estudo foi constatado que a adição de $0,5 \%$ de HPMC, acarretou no aumento da solubilidade aquosa do fármaco em até sete vezes, comparando com valores sem a adição do HPMC, que teve um incremento de quatro vezes na solubilidade do PZQ. Outra característica avaliada foi quanto a estabilidade, a adição do HPMC incrementou a constante de estabilidade do complexo Ciclodextrina/PZQ, aumentando a eficiência da complexação por otimizar a interação entre o fármaco e a $\beta C D$.

Outra inovação tecnológica considerada e estudada, é quanto a formação de sistemas de administração de fármacos baseados em implantes cilíndricos, utilizando polímeros biodegradáveis de poly( $\varepsilon$-caprolactone) (PCL) associado ao PZQ. O emprego desse polímero modifica o perfil de liberação do fármaco, na promoção de uma liberação prolongada (ZHANG, GUO, HUANG, 2009). No estudo feito por Li et al. (2010), na produção de implantes com um diâmetro de $8 \mathrm{~mm}$ portando de um carregamento com conteúdo de $25 \%$ de PZQ, promoveu um perfil de liberação mais lento do fármaco através da Lei de Fick, com uma liberação gradual do exterior do implante para o interior.

\section{CONCLUSÃO}

Portanto, é possível evidenciar o emprego da tecnologia farmacêutica no tratamento da esquistossomose, especialmente quando aplicada ao fármaco praziquantel, com o intuito de aprimorar suas propriedades biofarmacêuticas e melhorar sua eficácia. Todavia, o investimento 
realizado em pesquisas de inovações terapêuticas, por parte da indústria, para tratamento de tal parasitose, é mínimo, visto que é uma doença de caráter negligenciado e que acomete, majoritariamente, indivíduos pertencentes a uma classe socioeconômica desfavorecida. Logo, as inovações tecnológicas associadas ao PZQ na terapia esquistossomicida, são provenientes de estudos realizados, em sua maioria, por instituições acadêmicas, e que ainda estão em etapa de desenvolvimento.

\section{REFERÊNCIAS}

CUGOVCAN, M. et al. Biopharmaceutical characterization of praziquantel cocrystals and cyclodextrin complexes prepared by grinding. J. of Pharm. Biom. Ana, v. 137, p. 42-53, 2017.

DINORA, G. E. et al. In vitro characterization of some biopharmaceutical properties of praziquantel, Int. J. Pharm, v. 295, p. 93-99, 2005.

FREZZA, T. F., et al. Liposomal-praziquantel: Efficacy against Schistosoma mansoni in a preclinical assay. Acta Tropica, v. 128, p. 70-75, 2013.

LI, C. Effects of implant diameter, drug loading and end-capping on praziquantel release from PCL implants. Int. J. Pharm., v. 386, p. 23-29, 2010.

MOURÃO, L. C. S. et al. Effect of hydroxypropyl methylcellulose on beta cyclodextrin complexation of praziquantel in solution and in solid state. J. Incl. Phenom. Macrocycl. Chem., v. 85, p. 151-160, 2016.

RODRIGUES, S. G. Computational analysisand physico-chemical characterization of an inclusion compound between praziquantel and methyl-cyclodextrin for use as an alternative in the treatment of schistosomiasis, J. Incl. Phenom. Macrocycl. Chem., v. 70, p. 19-28, 2011.

SILVA, K. E. R. et al. Alternativas terapêuticas no combate à Esquistossomose Mansônica. Rev. de Ciênc. Farm. Bás. Apl., v. 33, n. 1, p. 9-16, 2012.

SILVA, V. B. R. et al. Medicinal chemistry of antischistosomal drugs: Praziquantel and oxamniquine. Bioorg. Medic. Chemis., v. 25, p. 3259-3277, 2017.

SOUZA, A. L. R. In vitro evaluation of permeation, toxicity and effect of praziquantel-loaded solid lipid nanoparticles against Schistosoma mansoni as a strategy to improve efficacy of the schistosomiasis treatment. Int. J. Pharm., v. 463, p. 31-37, 2014.

ZHANG, Y.; GUO, S.; HUANG, W. Tyrosine kinase inhibitor loaded PCL microspheres prepared by $\mathrm{S} / \mathrm{O} / \mathrm{W}$ technique using ethanol as pretreatment agent. Int. J. Pharm. 369, 1923, 2009. 